\title{
Epidemiological, Clinical and Pathological Features in Anticoagulant Rodenticide Toxicosis in Dogs: 66 Cases (2010-2015)
}

\author{
Andras-Laszlo NAGY*, Alexandru-Flaviu TABARAN, Roland BLAJ, Cornel CĂTOI, Alexandra POP, Adrian \\ Florin GAL, Adrian OROS \\ Faculty of Veterinary Medicine, University of Agricultural Sciences and Veterinary Medicine, 3-5 \\ Manasturstreet., 400372, Cluj-Napoca, Romania \\ *Corresponding author: nagyandras26@gmail.com
}

Bulletin UASVM Veterinary Medicine 72(2) / 2015,

Print ISSN 1843-5270; Electronic ISSN 1843-5378

DOI:10.15835/buasvmcn-vm: 11480

\begin{abstract}
Anticoagulant rodenticides are widely used pesticides globally. They act by inhibiting vitamin K epoxide reductase in the liver, decreasing the synthesis of clotting factors II, VII, IX, and X. Sixty six cases of anticoagulant rodenticide toxicosis were found during a retrospective study conducted at the Veterinary Hospital and the Pathology Department of the Faculty of Veterinary Medicine Cluj-Napoca, in a period between January 2010 and June 2015.The inclusion criteria were: clinical signs of exposure, coagulation testing result and typical pathological findings characteristic for bleeding syndrome. History, clinical and pathological data were collected from the medical records of the dogs presented to our units. In the present study, 66 cases of anticoagulant toxicosis in dogs were described, the animals ranging in age from 2 months to 12 years. 31 dogs were male and 35 female. Two cases followed exposure to brodifacoum, 4 cases exposure to warfarin and in the other cases the type of anticoagulant was unknown. The most common clinical signs were dyspnea, lethargy, weakness, anemia/palor, melena, spontaneous subcutaneous and muscular hematoma, sublingual hematoma and ecchymoses. The most important lesions were represented by haemothorax, thymic hematoma in juvenile dogs, subcutaneous and muscular hematomas, hemoperitoneum, severe anemia, and ecchymotic hemorrhages in different tissues. In cases were coagulation testing was performed, prolonged partial thromboplastin time, prothrombin time and coagulation time were recorded. Anticoagulant rodenticide toxicosis is frequently found in our region, the most important diagnostic tools being coagulation testing and toxicological exam.
\end{abstract}

Keywords: coagulopathy, retrospective, toxicology

\section{INTRODUCTION}

Anticoagulant rodenticides are widely used pesticides, poisonings with such substances being one of the most commonly encountered in veterinary practice. Anticoagulant substances, like Warfarin and its congeners are also used as therapeutic agents, (oral anticoagulants), overdose of these substances mimicking anticoagulant rodenticide poisoning (Gupta, 2007).

Both, first and second generation anticoagulants, act by inhibiting vitamin $\mathrm{K}$ epoxide reductase in the liver, decreasing the synthesis of clotting factors II, VII, IX, and X. By blocking the activity of vitamin K-epoxide reductase, the extrinsic, intrinsic, and common pathways of the coagulation cascade are blocked, resulting a coagulopathy (Sheafor, 1999).

\section{AIMS AND OBJECTIVES}

The aim of this study was to describe the epidemiological, clinical and pathological findings in anticoagulant rodenticide toxicosis in dogs in a retrospective study.

\section{MATERIALS AND METHODS}

Our study was conducted on sixty six cases of anticoagulant rodenticide toxicosis, identified during a retrospective study conducted at 


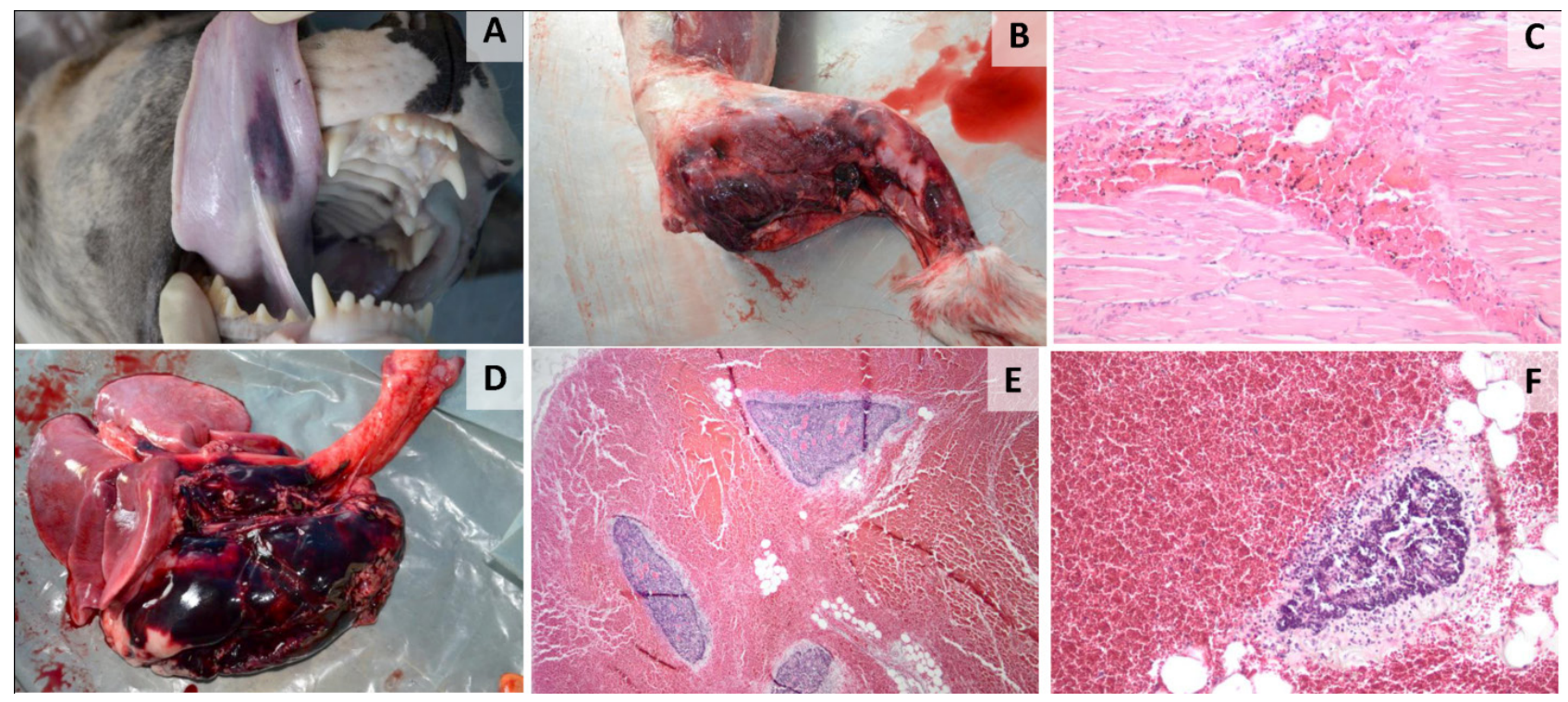

Fig.1. Sublingual hematoma (A),massive, diffuse muscular hemorrhages (B,C) massive timic hematomas in juvenile dogs (D), Thymus, disruption of normal lobular architecture, the interlobular septa are enlarged by the massive hemorrhage (E,F), HE x40 (E), HE x100 (C,F).

the Veterinary Hospital and the Pathology Department of the Faculty of Veterinary Medicine Cluj-Napoca, in a period between January 2010 and June 2015. The inclusion criteria were:clinical signs of anticoagulant toxicosis like coagulopathy, weakness, dyspnea; coagulation testing result and typical pathological findings characteristic for bleeding syndrome. History, clinical and pathological data were collected from the medical records of the dogs presented to our units. On cases presented for necropsy $(n=20)$ a complete necropsy and histological examination was realized using standard procedures.

\section{RESULTS AND DISCUSSION}

In our retrospective study 66 cases of anticoagulant toxicosis in dogs were described, the animals ranging in age from 2 months to 12 years. 31 dogs were male and 35 female. Two cases followed exposure to brodifacoum, 4 cases exposure to warfarin and in the other cases the type of anticoagulant was unknown. The most common clinical signs were dyspnea, lethargy, weakness, anemia/palor, melena, spontaneous subcutaneous and muscular hematoma, sublingual hematoma and ecchimoses in different tissues. The most important lesions were represented by haemothorax, thymic hematoma in juvenile dogs (Fig.1), subcutaneous and muscular hematomas (Fig.1), hemoperitoneum, severe anemia, and ecchimotic hemorrhages in different tissues. In cases were coagulation testing was performed, prolonged partial thromboplastin time, prothrombin time and coagulation time were recorded.

\section{CONCLUSION}

Anticoagulant rodenticide toxicosis is frequently found in dogs in our geographical region, clinical signs can guide the diagnosis, but the most important diagnostic tools are coagulation testing and toxicological exam.

Acknowledgements. This paper waspublished under the frame of European Social Found, Human Resources Development Operational Programme 2007-2013, project no. POSDRU/159/1.5/S/ 136893.

\section{REFERENCES}

1. Gupta RC (2007). Veterinary Toxicology, Basic and Clinical Principles, 2 ed. Academic Press, Elsevier, 557-559.

2. Sheafor S, Couto G (1999). Anticoagulant Rodenticide Toxicity in 21 Dogs. J Am Anim Hosp Assoc 35:38-46. 